\title{
DIFFERENTIAL EQUATIONS WITH A GIVEN SET OF SOLUTIONS
}

\author{
JAUME LLIBRE ${ }^{1}$, RAFAEL RAMÍREZ $^{2}$, AND NATALIA SADOVSKAIA ${ }^{3}$
}

\begin{abstract}
The aim of this paper is to study the following inverse problem of ordinary differential equations: For a given set of analytic functions $\omega=\left\{z_{1}(t), \ldots, z_{r}(t)\right\}$, with $z_{j}(t)=x_{j}(t)+i y_{j}(t)$ and $\bar{z}_{j}(t)=x_{j}(t)-i y_{j}(t)$ for $j=1, \ldots, r$, defined in the open interval $I \subseteq \mathbb{R}$, we want to determine the differential equation

$$
F\left(t, \bar{z}, z, \dot{z}, \dot{\bar{z}}, \ldots, z^{(n)}, \bar{z}^{(n)}\right)=0
$$

where $z^{(j)}=\frac{d^{j} z}{d t^{j}}$ for $j=1, \ldots, n$, in such a way that the given set of functions $\omega$ is a set of solutions of this differential equation.
\end{abstract}

\section{INTRODUCTION AND STATEMENT OF THE MAIN RESULTS}

In the theory of ordinary differential equations we can find two fundamental problems. The direct problem which consists in a broad sense in finding the solutions of a given ordinary differential equation, and the inverse problem. An inverse problem of ordinary differential equations is to find the more general differential equation satisfying a set of given properties (see $[4,10,19,20]$.

The inverse problem for determining the ordinary differential equations with given partial and first integrals was studied in [10]. The obtained results were applied in particular

(i) to construct Lagrangian mechanical systems with a given set of linear constraints with respect to the velocity and to obtain Hamiltonian systems with a given set of first integrals (see $[11,20]$ ),

(ii) to solve the 16th Hilbert problem for algebraic limit cycles (see [11, 12, 13, 14, 21]), and

(iii) to study the center-focus problem (see $[15,16,17])$.

In the rest of this paper we assume that all the functions are analytic in their variables, but we remark that this condition is for simplicity, although most of the results remain valid for $C^{r}$ functions with a convenient $r \geq 1$.

We study the following two problems on the determination of ordinary differential equations with a given set of solutions.

Problem I. Determine the most general ordinary differential equations of order $n$ of the form

$$
F\left(t, z, \dot{z}, \ldots, z^{(n)}\right)=0,
$$

for which a given set of functions $\omega=\left\{z_{1}(t), \ldots, z_{r}(t)\right\}$, with $z_{j}(t)=x_{j}(t)+i y_{j}(t)$ for $j=1, \ldots, r$, defined in the open interval $I \subseteq \mathbb{R}$ are solutions of the equation (1).

We consider the following particular case of Problem I.

Key words and phrases. planar differential system, inverse problem for ordinary differential equations, Ricatti equation, Abel equation, first integral. 
Problem I.1 Determine the conditions on the set of functions $\omega=\left\{z_{1}(t), \ldots, z_{k}(t)\right\}$ in order that a differential equation of the form

$$
\dot{z}=f(t, z) \text {, }
$$

has first the functions $z_{j}(t)$, for $j=1, \ldots, k$, as solutions, and second an invariant of the form

$$
F=e^{\Psi} \Lambda(t) \prod_{j=1}^{k}\left(z-z_{j}(t)\right)^{\alpha_{j}}\left(\bar{z}-\bar{z}_{j}(t)\right)^{\gamma_{j}},
$$

where $\Psi=\Psi(z, \bar{z}, t)$ and $\Lambda=\Lambda(t)$ are functions, and $\alpha_{j}$ and $\gamma_{j}$, are complex constants for $j=1, \ldots, k$.

Problem I.1 is studied in sections 4 and 5 .

Problem II. Determine the most general ordinary differential equations of order $n$

$$
F\left(t, \bar{z}, z, \dot{z}, \dot{\bar{z}}, \ldots, z^{(n)}, \bar{z}^{(n)}\right)=0
$$

for which the given set of functions $\omega=\left\{z_{1}(t), \ldots, z_{r}(t)\right\}$, with $z_{j}(t)=x_{j}(t)+i y_{j}(t)$ and $\bar{z}_{j}(t)=x_{j}(t)-i y_{j}(t)$ for $j=1, \ldots, r$, defined in the open interval $I \subseteq \mathbb{R}$ are solutions of the equation (4). We shall study the following particular case of Problem II.

\section{Problem II.1 Let}

$$
\dot{z}=F(t, z, \bar{z}),
$$

be a differential system, where $F(t, z, \bar{z})$ is a polynomial of degree $n$ in the variables $z$ and $\bar{z}$.

(i) Determine the conditions of the set of functions $\omega=\left\{z_{1}(t), \ldots, z_{k}(t)\right\}$ for $k \geq 1$, in order that there exists a differential equation (5) having the set of functions $\omega$ as solutions.

(ii) Determine the maximum numbers of functions $z_{j}(t)$ for $j=1, \ldots, k$, which define a unique polynomial differential system (5).

Problem II.1 is study in section 6 .

The main results are the following.

Theorem 1. Let $\omega=\left\{z_{1}(t), \ldots, z_{n}(t)\right\}$ be a set of functions such that

$$
\operatorname{det}(A):=\Delta(\omega)=\left|\begin{array}{cccc}
z_{1}(t) & z_{2}(t) & \ldots & z_{n}(t) \\
\dot{z}_{1}(t) & \dot{z}_{2}(t) & \ldots & \dot{z}_{n}(t) \\
\vdots & \vdots & \ldots & \vdots \\
z_{1}^{(n-1)}(t) & z_{2}^{(n-1)}(t) & \ldots & z_{n}^{(n-1)}(t)
\end{array}\right| \neq 0 .
$$

Then the most general linear ordinary differential equations of order $n$ of the form

$$
a_{0}(t) z^{(n)}+\ldots+a_{n-1}(t) \dot{z}+a_{n}(t) z=0, \quad a_{0}(t) \neq 0
$$

having $\omega$ as a set of solutions is

$$
W\left(z, z_{1}(t), \ldots, z_{n}(t)\right)=\left|\begin{array}{ccccc}
z & z_{1}(t) & z_{2}(t) & \ldots & z_{n}(t) \\
\dot{z} & \dot{z}_{1}(t) & \dot{z}_{2}(t) & \ldots & \dot{z}_{n}(t) \\
\vdots & \vdots & \vdots & \ldots & \vdots \\
z^{(n)} & z_{1}^{(n)}(t) & z_{2}^{(n)}(t) & \ldots & z_{n}^{(n)}(t)
\end{array}\right|=0 .
$$

Moreover $z(t)=\sum_{j=1}^{n} C_{j} z_{j}(t)$ is a general solution of this differential equation, where $C_{j}$ are arbitrary constants. 
Corollary 2. If the set of functions $\omega=\left\{z_{1}(t), \ldots, z_{n}(t)\right\}$ satisfies $(6)$, then the general solution of the differential equation

$$
a_{0}(t) z^{(n)}+\ldots+a_{n}(t) \dot{z}+a_{n}(t) z=f(t), \quad a_{0}(t) \neq 0
$$

is

$$
z=C_{1}(t) z_{1}(t)+C_{2}(t) z_{2}(t)+\ldots+C_{n}(t) z_{n}(t),
$$

where $C_{1}(t), \ldots, C_{n}(t)$ are functions such that $A \dot{C}=\Lambda$, where $C=\left(C_{1}, \ldots, C_{n}\right)^{T}, \Lambda=$ $(0, \ldots, 0, f(t))^{T}$, and the matrix $A$ is defined in $(6)$.

Theorem 1 and Corollary 2 are proved in section 2 .

Theorem 3. Let $\omega=\left\{z_{1}(t), \ldots, z_{n+1}(t)\right\}$ be a set of functions such that the Vandermonde determinant

(8)

$$
\triangle(\omega)=\left|\begin{array}{ccccc}
z_{1}^{n}(t) & z_{1}^{n-1}(t) & \ldots & z_{1}(t) & 1 \\
z_{2}^{n}(t) & z_{2}^{n-1}(t) & \ldots & z_{2}(t) & 1 \\
z_{3}^{n}(t) & z_{3}^{n-1}(t) & \ldots & z_{3}(t) & 1 \\
\vdots & \vdots & \ldots & \vdots & \vdots \\
z_{n+1}^{n}(t) & z_{n+1}^{n-1}(t) & \ldots & z_{n+1}(t) & 1
\end{array}\right|:=\operatorname{det} A=\prod_{1 \leq k \leq j \leq n}\left(z_{k}(t)-z_{j}(t)\right) \neq 0, .
$$

and $\sum_{j=1}^{n+1}\left|\dot{z}_{j}\right|^{2} \neq 0$.

Then any first order nonlinear ordinary differential equation of the form

$$
a_{0}(t) z^{m} \dot{z}+a_{1}(t) z^{n}+\ldots+a_{n-1}(t) z^{2}+a_{n}(t) z+a_{n+1}(t)=0,
$$

where $n$ and $m$ are non-negative integers with $a_{0}(t) \neq 0$ and having $z_{1}(t), \ldots, z_{n+1}(t)$ as solutions can be written as

$$
\left|\begin{array}{ccccc}
1 & 1 & 1 & \ldots & 1 \\
z & z_{1}(t) & z_{2}(t) & \ldots & z_{n+1}(t) \\
z^{2} & z_{1}^{2}(t) & z_{2}^{2}(t) & \ldots & z_{n+1}^{2}(t) \\
\vdots & \vdots & \vdots & \ldots & \vdots \\
z^{n} & z_{1}^{n}(t) & z_{2}^{n}(t) & \ldots & z_{n+1}^{n}(t) \\
z^{m} \dot{z} & z_{1}^{m}(t) \dot{z}_{1}(t) & z_{2}^{m}(t) \dot{z}_{2}(t) & \ldots & z_{n+1}^{m}(t) \dot{z}_{n+1}(t)
\end{array}\right|:=z^{m} \triangle(\omega) \dot{z}-Z(z, t)=0
$$

Moreover if $z_{j}=$ constant for $j=1, \ldots, n$ then there exist the invariant

$$
\int\left(\frac{z^{m} d z(t)}{\prod_{j=1}^{n}\left(z(t)-z_{j}\right)}-\frac{z_{n+1}^{m}(t) d z_{n+1}(t)}{\prod_{j=1}^{n}\left(z_{n+1}(t)-z_{j}\right)}\right)
$$

which is constant on the solutions of (9).

Corollary 4. Differential equation (10) for $m=0$ under the change

can be written as

$$
z=z_{n+1}+\frac{1}{w}
$$

$$
w^{n-2} \dot{w}=\beta_{1}(t) w^{n-1}+\beta_{2}(t) w^{n-2}+\ldots+\beta_{n}(t),
$$

where $\left.\frac{\partial^{j} Z(z, t)}{\partial z^{j}}\right|_{z=z_{n+1}}=-\beta_{j}(t)$, for $j=1, \ldots, n$, where $Z(z, t)$ is defined in $(10)$. 
Theorem 3 and Corollary 4 are proved in section 3 .

The differential equation

$$
z^{\prime}=p(t) z^{3}+q(t) z^{2}+r(t) z+s(t)=Q(t, z), \quad \text { with } \quad p(t) \neq 0,
$$

is called the Abel equation of first kind and the differential equation

$$
w w^{\prime}+\Psi(t) w=\Phi w^{2}+\Upsilon(t),
$$

is called the Abel equation of a second kind. Note that $\Upsilon(t) \neq 0$, otherwise we have a linear differential equation.

The Abel equations in general has no invariants, but there exist some particular cases for which these equations have an invariant. We shall study some of these cases.

Proposition 5. Let $w_{j}=w_{j}(t)$ for $j=1,2,3$ be solutions of the Abel differential equation of second kind (14). Then equation (14) has the invariant

$$
F=e^{-\sum_{j=1}^{3} m_{j} \int \Phi(t) d t} \prod_{j=1}^{3}\left(w-w_{j}(t)\right)^{m_{j}}
$$

if and only if

$$
\sum_{j=1}^{3} \frac{m_{j}}{w_{j}(t)}=0
$$

Here the $m_{j}$ are constants not all zero.

Corollary 6. The Abel differential equation of the first kind has the invariant

$$
F=e^{-\sum_{j=1}^{3} m_{j} \int \Phi(t) d t} \prod_{j=1}^{3}\left(\frac{z-z_{j}}{\left(z-z_{4}\right)\left(z_{j}-z_{4}\right)}\right)^{m_{j}}:=\Lambda(t) \prod_{j=1}^{3}\left(\frac{z-z_{j}}{z-z_{4}}\right)^{m_{j}},
$$

if and only if

$$
\begin{gathered}
\left.\sum_{j=1}^{3} m_{j}\left(z_{j}-z_{4}\right)\right)=0 . \\
\text { if } \sum_{j=1}^{3} m_{j}=0 \text { then } \sum_{j=1}^{3} m_{j} z_{j}=0 \text { and the invariant } F \text { becomes } \\
F=\prod_{k=1, k \neq 4}^{3}\left(\frac{z-z_{j}}{z_{j}-z_{4}}\right)^{m_{j}}=\Lambda(t) \prod_{k=1, k \neq 4}^{3}\left(z-z_{j}\right)^{m_{j}},
\end{gathered}
$$

Moreover

Proposition 5 and Corollary 6 are proved in section 5 . 
Proposition 7. Let $\omega_{4}=\left\{z_{1}(t), \ldots, z_{\kappa}(t)\right\}$ for $\kappa=(m+1)(m+2) / 2$ be a set of functions such that

$$
\Delta(\omega):=\left|\begin{array}{cccc}
1 & 1 & \cdots & 1 \\
z_{1} & z_{2} & \ldots & z_{\kappa} \\
\bar{z}_{1} & \bar{z}_{2} & \ldots & \bar{z}_{\kappa} \\
\vdots & \vdots & \ldots & \vdots \\
z_{1}^{m} & z_{2}^{m} & \ldots & z_{\kappa}^{m} \\
\vdots & \vdots & \ldots & \vdots \\
z_{1} \bar{z}_{1}^{m-1} & z_{2}(t) \bar{z}_{2}^{m-1} & \ldots & z_{\kappa} \bar{z}_{\kappa}^{m-1}(t) \\
\bar{z}_{1}^{m} & \bar{z}_{2}^{m} & \ldots & \bar{z}_{\kappa}^{m}
\end{array}\right| \neq 0 \text { with } \kappa=(m+1)(m+2) / 2
$$

as solutions can be written as

$$
\left|\begin{array}{ccccc}
1 & 1 & 1 & \ldots & 1 \\
z & z_{1} & z_{2} & \ldots & z_{\kappa} \\
\bar{z} & \bar{z}_{1} & \bar{z}_{2} & \ldots & \bar{z}_{\kappa} \\
\vdots & \vdots & \vdots & \ldots & \vdots \\
z^{m} & z_{1}^{m} & z_{2}^{m} & \ldots & z_{\kappa}^{m} \\
\vdots & \vdots & \vdots & \ldots & \vdots \\
z \bar{z}^{m-1} & z_{1} \bar{z}_{1}^{m-1} & z_{2} \bar{z}_{2}^{m-1} & \ldots & z_{\kappa} \bar{z}_{\kappa}^{m-1} \\
\bar{z}^{m} & \bar{z}_{1}^{m} & \bar{z}_{2}^{m} & \ldots & \bar{z}_{\kappa}^{m} \\
\dot{z} & \dot{z}_{1} & \dot{z}_{2} & \ldots & \dot{z}_{\kappa}
\end{array}\right|=0
$$

Moreover if $z_{j}=$ constant for $j=1, \ldots, \kappa-1$ then there exist the invariant

$$
\int\left(\frac{d z}{\Upsilon\left(z, z_{1}, \ldots, z_{\kappa-1}\right)}-\frac{d z_{\kappa}}{\Upsilon\left(z_{\kappa}, z_{1}, \ldots, z_{\kappa-1}\right)}\right)=C=\text { constant } .
$$

where

$$
\Upsilon\left(\xi, z_{1}, \ldots, z_{\kappa-1}\right)=\left|\begin{array}{ccccc}
1 & 1 & 1 & \ldots & 1 \\
\xi & z_{1} & z_{2} & \ldots & z_{\kappa-1} \\
\bar{\xi} & \bar{z}_{1} & \bar{z}_{2} & \ldots & \bar{z}_{\kappa-1} \\
\vdots & \vdots & \vdots & \ldots & \vdots \\
\xi^{m} & z_{1}^{m} & z_{2}^{m} & \ldots & z_{\kappa-1}^{m} \\
\vdots & \vdots & \vdots & \ldots & \vdots \\
\xi \bar{\xi}^{m-1} & z_{1} \bar{z}_{1}^{m-1} & z_{2} \bar{z}_{2}^{m-1} & \ldots & z_{\kappa-1} \bar{z}_{\kappa-1}^{m-1} \\
\bar{\xi}^{m} & \bar{z}_{1}^{m} & \bar{z}_{2}^{m} & \ldots & \bar{z}_{\kappa-1}^{m}
\end{array}\right|
$$

Proposition 7 is proved in section 6 . 
Proposition 8. Consider the differential equation of the form

$$
\dot{z}=\sum_{n=0}^{m} \sum_{j+k=n} a_{j k}(t) z^{j} \bar{z}^{k}
$$

with $m+1$ complex solutions $\omega=\left(z_{1}(t), \ldots, z_{m+1}(t)\right)$ such that

$$
\bar{z}_{j}(t)=e^{i \alpha} z_{j}(t), \quad \text { for } \quad j=1, \ldots, m+1,
$$

where $\alpha \in \mathbb{R}$ and the Vandermonde determinant

$$
\Delta(\omega)=\left|\begin{array}{ccccc}
1 & 1 & 1 & \ldots & 1 \\
z_{1} & z_{2} & z_{3} & \ldots & z_{m+1} \\
z_{1}^{2} & z_{2}^{2} & z_{3}^{2} & \ldots & z_{m+1}^{2} \\
\vdots & \vdots & \vdots & \ldots & \vdots \\
z_{1}^{m} & z_{1}^{m} & z_{1}^{m} & \ldots & z_{m+1}^{m}
\end{array}\right| \neq 0
$$

can be written as

$$
\frac{1}{\Delta(\omega)}\left|\begin{array}{cccccc}
1 & 1 & 1 & 1 & \ldots & 1 \\
z & z_{1} & z_{2} & z_{3} & \ldots & z_{m+1} \\
z^{2} & z_{1}^{2} & z_{2}^{2} & z_{3}^{2} & \ldots & z_{m+1}^{2} \\
\vdots & \vdots & \vdots & \vdots & \ldots & \vdots \\
z^{m} & z_{1}^{m} & z_{1}^{m} & z_{1}^{m} & \ldots & z_{m+1}^{m} \\
\dot{z} & \dot{z}_{1} & \dot{z}_{2} & \dot{z}_{3} & \ldots & \dot{z}_{m+1}
\end{array}\right|=\left(\bar{z}-e^{i \alpha} z\right) P(z, \bar{z}, t)
$$

where $P(z, \bar{z}, t)$ is a convenient polynomial of degree $m-1$.

Proposition 8 is proved in subsection 6.2 .

\section{Proofs of Theorem 1 And Corollary 2}

Proof of Theorem 1. Clearly differential system (7) has the solutions given by the set of functions $\omega$. Indeed, if $z=z_{j}(t)$ with $j=1, \ldots, n$, then in view of the properties of the determinant we have that $W\left(z_{j}(t), z_{1}(t), \ldots, z_{n}(t)\right) \equiv 0$

The reciprocity is proved as follows. We assume that we have the linear differential equation of order $n$

$$
a_{0}(t) z^{(n)}+\ldots+a_{n-1}(t) \dot{z}+a_{n}(t) z=0, \quad a_{0}(t) \neq 0
$$

and let $\omega=\left\{z_{1}(t), \ldots, z_{n}(t)\right\}$ be the set of functions satisfying (6). Then, solving the system

$$
a_{0}(t) z_{j}^{(n)}+\ldots+a_{n-1}(t) \dot{z}_{j}+a_{n}(t) z_{j}=0, \quad \text { for } \quad j=1, \ldots, n
$$


with respect to $a_{1}, \ldots, a_{n}$ after some computations we get that

$$
\begin{aligned}
& a_{1}(t)=\frac{(-1)^{n+2} a_{0}(t)}{\Delta(\omega)}\left|\begin{array}{cccc}
z_{1}(t) & z_{2}(t) & \ldots & z_{n}(t) \\
\dot{z}_{1}(t) & \dot{z}_{2}(t) & \ldots & \dot{z}_{n}(t) \\
\vdots & \vdots & \ldots & \vdots \\
z_{1}^{(n-2)}(t) & z_{2}^{(n-2)}(t) & \ldots & z_{n}^{(n-2)}(t) \\
z_{1}^{(n)}(t) & z_{2}^{(n)}(t) & \ldots & z_{n}^{(n)}(t)
\end{array}\right|:=\frac{(-1)^{n+2} a_{0}(t) \Delta_{1}(\omega)}{\Delta(\omega)} \\
& a_{n}(t)=\frac{-a_{0}(t)}{\Delta(\omega)}\left|\begin{array}{cccc}
\dot{z}_{1}(t) & \dot{z}_{2}(t) & \ldots & \dot{z}_{n}(t) \\
\ddot{z}_{1}(t) & \ddot{z}_{2}(t) & \ldots & \ddot{z}_{n}(t) \\
\vdots & \vdots & \ldots & \vdots \\
z_{1}^{(n)}(t) & z_{2}^{(n)}(t) & \ldots & z_{n}^{(n)}(t)
\end{array}\right|:=\frac{-a_{0}(t) \Delta_{n}(\omega)}{\Delta(\omega)}
\end{aligned}
$$

Inserting $a_{1}, \ldots, a_{n}$ in $(7)$ we obtain

$$
\frac{a_{0}(t)}{\Delta(\omega)}\left(\Delta(\omega) z^{(n)}+(-1)^{n+2} \Delta_{1}(\omega) z^{(n-1)}+\ldots-\Delta_{n}(\omega) z\right)=0 .
$$

This last expression is the development of the determinant given in (8) using its first column. Hence the theorem is proved.

Proof of Corollary 2. The proof of these Theorem is well known as variation of constants, is a general method to solve inhomogeneous linear ordinary differential equations.

\section{Proofs of Theorem 3 and Corollary 4}

Proof of Theorem 3. First we prove that the set of function $\omega=\left\{z_{1}(t), \ldots, z_{n+1}(t)\right\}$ satisfies (10). Indeed, in view of the properties of the determinant we get that

$$
\left|\begin{array}{ccccc}
1 & 1 & 1 & \ldots & 1 \\
z_{j}(t) & z_{1}(t) & z_{2}(t) & \ldots & z_{n+1}(t) \\
z_{j}^{2}(t) & z_{1}^{2}(t) & z_{2}^{2}(t) & \ldots & z_{n+1}^{2}(t) \\
\vdots & \vdots & \vdots & \ldots & \vdots \\
z_{j}^{n}(t) & z_{1}^{n}(t) & z_{2}^{n}(t) & \ldots & z_{n+1}^{n}(t) \\
\dot{z}_{j}(t) & \dot{z}_{1}(t) & \dot{z}_{2}(t) & \ldots & \dot{z}_{n+1}(t)
\end{array}\right|=0
$$

for $j=1, \ldots, n+1$.

The reciprocity is proved as follows. We assume that we have the nonlinear differential equation of first order $(10)$, and let $\omega=\left\{z_{1}(t), \ldots, z_{n+1}(t)\right\}$ be the set of functions satisfying (8). Then from the relations

$$
a_{1}(t) z_{j}^{n}+\ldots+a_{n-1}(t) z_{j}^{2}+a_{n}(t) z_{j}+a_{n+1}(t)=-a_{0}(t) z_{j}^{m} \dot{z}_{j} . \quad \text { for } \quad j=1, \ldots, n+1,
$$

Since this system is a linear system with respect to $a_{1}(t), \ldots, a_{n+1}(t)$ it can be written in the matrix form

$$
\left(\begin{array}{ccccc}
z_{1}^{n}(t) & z_{1}^{n-1}(t) & \ldots & z_{1}(t) & 1 \\
z_{2}^{n}(t) & z_{2}^{n-1}(t) & \ldots & z_{2}(t) & 1 \\
z_{3}^{n}(t) & z_{3}^{n-1}(t) & \ldots & z_{3}(t) & 1 \\
\vdots & \vdots & \ldots & \vdots & \vdots \\
z_{n+1}^{n}(t) & z_{n+1}^{n-1}(t) & \ldots & z_{n+1}(t) & 1
\end{array}\right)\left(\begin{array}{c}
a_{1}(t) \\
a_{2}(t) \\
a_{3}(t) \\
\vdots \\
a_{n+1}(t)
\end{array}\right)=-a_{0}\left(\begin{array}{c}
z_{1}^{m}(t) \dot{z}_{1}(t) \\
z_{2}^{m}(t) \dot{z}_{2}(t) \\
z_{1}^{m}(t) \dot{z}_{3}(t) \\
\vdots \\
z_{n+1}^{m}(t) \dot{z}_{n+1}(t)
\end{array}\right)
$$


In view of condition (8) and by applying the Cramer's rule we obtain that

$$
\begin{gathered}
a_{1}(t)=-\frac{a_{0}(t)}{\Delta(\omega)}\left|\begin{array}{ccccc}
z_{1}^{m}(t) \dot{z}_{1}(t) & z_{1}^{n-1}(t) & \ldots & z_{1}(t) & 1 \\
z_{2}^{m}(t) \dot{z}_{2}(t) & z_{2}^{n-1}(t) & \ldots & z_{2}(t) & 1 \\
z_{3}^{m}(t) \dot{z}_{3}(t) & z_{3}^{n-1}(t) & \ldots & z_{3}(t) & 1 \\
\vdots & \vdots & \ldots & \vdots & \vdots \\
z_{n+1}^{m}(t) \dot{z}_{n+1}(t) & z_{n+1}^{n-1}(t) & \ldots & z_{n+1}(t) & 1
\end{array}\right|:=-\frac{a_{0}(t) \Delta_{1}(\omega)}{\Delta(\omega)}, \\
\vdots \\
\vdots
\end{gathered}
$$

By inserting $a_{1}(t), \ldots, a_{n+1}(t)$ into (9) after some computations we get (10).

$$
\frac{a_{0}(t)}{\Delta(\omega)}\left(\Delta(\omega) z^{m} z^{(n)}+(-1)^{n+2} \Delta_{1}(\omega) z^{(n-1)}+\ldots-\Delta_{n}(\omega) z\right)=0 .
$$

This last expression is the development of the determinant given in 10 using its first column. To prove the relation (11) it is easy to obtain by considering that $\dot{z}_{j}=0$ for $j=1, \ldots, n$.

Clearly if $\sum_{j=1}^{n+1}\left|\dot{z}_{j}\right|^{2}=0$ then the all coefficients $a_{j}(t)$ for $j=1, \ldots, n+1$ are equal to zero. Consequently differential system (9) becomes $\dot{z}=0$. Thus the theorem is proved.

Proof of Corollary 4. By developing the polynomial $Z(z, t)$ of degree $n$ in the variable $z$ (see formula (10)) in powers of $z-z_{n+1}$ we get

$$
\begin{aligned}
Z(z, t)= & Z\left(z_{n+1}, t\right)+\left.\frac{\partial Z(z, t)}{\partial z}\right|_{z=z_{n+1}}\left(z-z_{n+1}\right)+\left.\frac{1}{2 !} \frac{\partial^{2} Z(z, t)}{\partial z^{2}}\right|_{z=z_{n+1}}\left(z-z_{n+1}\right)^{2} \\
& +\ldots+\left.\frac{1}{n !} \frac{\partial^{n} Z(z, t)}{\partial z^{n}}\right|_{z=z_{n+1}}\left(z-z_{n+1}\right)^{n} \\
= & Z\left(z_{n+1}, t\right)-\beta_{1}(t)\left(z-z_{n+1}\right)-\beta_{2}(t)\left(z-z_{n+1}\right)^{2}-\ldots-\beta_{n}(t)\left(z-z_{n+1}\right)^{n} .
\end{aligned}
$$

Introducing the new variable $1 / w=z-z_{n+1}$ and by considering that $\Delta(\omega) \dot{z}_{n+1}=Z\left(z_{n+1}, t\right)$ after some computations we obtain (12). Then the corollary is proved.

Example 1. For differential equation (10) for $m=0$ and $n=2$ (Ricatti equation)

$$
\begin{aligned}
z^{\prime}= & \frac{z_{1}^{\prime}\left(z_{3}-z_{2}\right)+z_{2}^{\prime}\left(z_{1}-z_{3}\right)+z_{3}^{\prime}\left(z_{2}-z_{1}\right)}{\Delta(\omega)} z^{2} \\
& -\frac{z_{1}^{\prime}\left(z_{3}^{2}-z_{2}^{2}\right)+z_{2}^{\prime}\left(z_{1}^{2}-z_{3}^{2}\right)+z_{3}^{\prime}\left(z_{2}^{2}-z_{1}^{2}\right)}{\Delta(\omega)} z \\
& +\frac{z_{1}^{\prime}\left(z_{3}-z_{2}\right) z_{3} z_{2}+z_{2}^{\prime}\left(z_{1}-z_{3}\right) z_{1} z_{3}+z_{3}^{\prime}\left(z_{2}-z_{1}\right) z_{2} z_{1} .}{\Delta(\omega)} .
\end{aligned}
$$

differential equation (12) becomes

$$
\dot{w}=\beta_{1}(t) w+\beta_{2}(t), \quad w=\frac{1}{z-z_{3}}
$$


with

$$
\begin{aligned}
& \beta_{1}(t)=\frac{\left(z_{2}-z_{3}\right) \dot{z}_{1}}{\left(z_{1}-z_{3}\right)\left(z_{1}-z_{2}\right)}-\frac{\left(z_{1}-z_{3}\right) \dot{z}_{2}}{\left(z_{2}-z_{3}\right)\left(z_{1}-z_{2}\right)}+\frac{\left(z_{1}-2 z_{3}+z_{2}\right) \dot{z}_{3}}{\left(z_{2}-z_{3}\right)\left(z_{1}-z_{3}\right)} \\
& \beta_{2}(t)=-\frac{\dot{z}_{1}}{\left(z_{1}-z_{3}\right)\left(z_{1}-z_{2}\right)}+\frac{\dot{z}_{2}}{\left(z_{2}-z_{3}\right)\left(z_{1}-z_{2}\right)}-\frac{\dot{z}_{3}}{\left(z_{2}-z_{3}\right)\left(z_{1}-z_{3}\right)}
\end{aligned}
$$

Example 2. For differential equation (10) for $m=0$ and $n=3$ (Abel equation of first kind), having four solutions $z_{1}, z_{2}, z_{3}, z_{4}$ satisfying (8) can be written as (10) or equivalently

$$
z^{\prime}=\frac{\Delta_{1}(\omega)}{\Delta(\omega)} z^{3}-\frac{\Delta_{2}(\omega)}{\Delta(\omega)} z^{2}+\frac{\Delta_{3}(\omega)}{\Delta(\omega)} z-\frac{\Delta_{4}(\omega)}{\Delta(\omega)}:=Z_{0}(z, t) .
$$

equation (12) becomes

$$
w \dot{w}=\beta_{1}(t) w^{2}+\beta_{2}(t) w+\beta_{3}(t), \quad w=\frac{1}{z-z_{4}}
$$

with

$$
\begin{aligned}
\beta_{1}(t)= & \lambda\left(z_{1}, z_{2}, z_{3}, z_{4}\right) \dot{z}_{1}+\lambda\left(z_{2}, z_{1}, z_{3}, z_{4}\right) \dot{z}_{2}+\lambda\left(z_{1}, z_{3}, z_{2}, z_{4}\right) \dot{z}_{3} \\
& +\frac{\left(z_{2}-z_{4}\right)\left(z_{1}-z_{4}\right)+\left(z_{3}-z_{4}\right)\left(z_{1}-z_{2}-2 z_{4}\right)}{\left(z_{1}-z_{4}\right)\left(z_{3}-z_{4}\right)\left(z_{2}-z_{4}\right)} \dot{z}_{4}, \\
\beta_{2}(t)= & \mu\left(z_{1}, z_{2}, z_{3}, z_{4}\right) \dot{z}_{1}+\mu\left(z_{2}, z_{1}, z_{3}, z_{4}\right) \dot{z}_{2}+\mu\left(z_{1}, z_{3}, z_{2}, z_{4}\right) \dot{z}_{3}+\mu\left(z_{1}, z_{2}, z_{4}, z_{3}\right) \dot{z}_{4}, \\
\beta_{3}(t)= & \nu\left(z_{1}, z_{2}, z_{3}, z_{4}\right) \dot{z}_{1}+\nu\left(z_{2}, z_{1}, z_{3}, z_{4}\right) \dot{z}_{2}+\nu\left(z_{1}, z_{3}, z_{2}, z_{4}\right) \dot{z}_{3}+\nu\left(z_{1}, z_{2}, z_{4}, z_{3}\right) \dot{z}_{4},
\end{aligned}
$$

where $\lambda(u, v, w, r)=-\frac{(w-r)(v-u)}{(u-v)(u-w)(u-r)}, \quad \mu(u, v, w, r)=\frac{v-2 r+w}{(u-v)(u-w)(u-r)}$, and $\nu(u, v, w, r)=-\frac{1}{(u-v)(u-w)(u-r)}$.

We say that the set of functions $\omega_{1}=\left\{z_{1}, z_{2}, \ldots, z_{n+1}\right\}$ which are solutions of equation (10) and the set of functions $\omega_{\kappa}=\left\{z_{1}^{(\kappa)}, \ldots, z_{n+1}^{(\kappa)}\right\}$ are equivalent and we write $\omega_{1} \simeq \omega_{\kappa}$ if

$$
\frac{\Delta_{j}(\omega)}{\Delta(\omega)}=\frac{\Delta_{j}\left(\omega_{\kappa}\right)}{\Delta\left(\omega_{\kappa}\right)}, \quad \text { for } \quad j=1, \ldots, n+1 \quad \kappa \in \mathbb{N} .
$$

Clearly if $\omega_{1} \simeq \omega_{\kappa}$ then $\tilde{\omega}_{1} \simeq \tilde{\omega}_{\kappa}$ where $\tilde{\omega}_{1}=\left\{z_{1}, z_{2}, z_{j-1}, z_{j}^{(\kappa)}, z_{j+1}, \ldots, z_{n+1}\right\}$ and $\tilde{\omega}_{\kappa}=$ $\left\{z_{1}^{(\kappa)}, \ldots, z_{j-1}^{(\kappa)}, z_{j}, z_{j+1}^{(\kappa)}, \ldots, z_{n+1}^{(\kappa)},\right\}$, The set of equivalent solutions of (10) we shall denote by $\Omega$.

Example 3. For the Abel differential equations of the first kind

$$
\dot{z}=\frac{225 z^{3}+16\left(3 t^{8}-17 t^{6}+6 t^{4}-1\right) z}{4 t\left(t^{4}-1\right)\left(t^{4}-4\right)\left(4 t^{4}-1\right)}
$$

a set of equivalent solutions $\Omega$ is (see [6])

$$
\left\{z_{1}=-z_{2}=\frac{2}{5} t\left(t^{2}+1\right), \quad z_{3}=-z_{4}=\frac{2}{3} t\left(t^{2}-1\right), \quad z_{5}=-z_{6}=\frac{4}{15}\left(t^{4}-1\right), \quad z_{7}=0\right\}
$$

\section{Study of Problem I.1}

4.1. Nonlinear differential equations with constant solutions. Problem I.1 has an easy solution when the functions $z_{j}(t)$, for $j=1, \ldots, n+1$, are complex constant $z_{j}$, because 
then $f(t, z)=\lambda(t) \prod_{s=1}^{n+1}\left(z-z_{s}\right)^{\kappa_{s}}$ with $\kappa_{j} \in \mathbb{N}$ and $\lambda(t)$ is a convenient function. Indeed, from (9) it follows that

$$
\dot{z}=\lambda(t) \prod_{s=1}^{n+1}\left(z-z_{s}\right)^{\kappa_{s}}=\lambda(t) g(z)
$$

where for simplicity we assume that the function $\lambda(t)$ is real. From the equations $\dot{z}=\lambda(t) g(z)$ and $\dot{\bar{z}}=\lambda(t) g(\bar{z})$ it follows that

$$
\frac{d z}{g(z)}-\frac{d \bar{z}}{g(\bar{z})}=0
$$

After the integration of (29) we get that

$$
\tilde{F}=\int\left(\frac{d z}{f(z)}-\frac{d \bar{z}}{f(\bar{z})}\right)
$$

is a first integral of the differential equation (29). It is possible to show that the function $F=\exp \tilde{F}$ is an invariant, that in this case is a first integral

$$
F=e^{\Psi} \frac{\prod_{s=1}^{n+1}\left(z-z_{s}\right)^{A_{s}^{(1)}}}{\prod_{s=1}^{n+1}\left(\bar{z}-\bar{z}_{s}\right)^{\bar{A}_{s}^{(1)}}}
$$

where

$$
\begin{aligned}
\Psi:= & \left(-\frac{A_{1}^{(2)}}{\left(z-z_{1}\right)}+\ldots+\frac{1}{\left(1-\kappa_{1}\right)} \frac{A_{1}^{\left(k_{s}\right)}}{\left(z-z_{1}\right)^{-1+\kappa_{1}}}\right)+\ldots \\
& -\left(-\frac{\bar{A}_{n+1}^{(2)}}{\bar{z}-\bar{z}_{n+1}}+\ldots+\frac{1}{\left(1-\kappa_{n+1}\right)} \frac{\bar{A}_{n+1}^{\left(k_{n+1}\right)}}{\left(\bar{z}-\bar{z}_{n+1}\right)^{-1+\kappa_{n+1}}}\right),
\end{aligned}
$$

where $A_{m}^{(j)}$ is a convenient constants for $j=2, \ldots, \kappa_{n+1}$ and $m=1, \ldots, n+1$. In short, Problem I.1 is solved when all the functions of $\omega$ are constant.

4.2. First order linear ordinary differential equations and Riccati equations. Here we see that Problem I.1 is easy to solve for first order linear ordinary differential equations and for the Riccati equations. Indeed, by Theorem 3 the first order linear differential equation with the two solutions $z_{1}(t)$ and $z_{2}(t)$ such that $\Delta(\omega)=z_{1}(t)-z_{2}(t) \neq 0$, can be written as (10) for $n=1$ and $m=0$ is

$$
z^{\prime}-\frac{\left.z_{2}^{\prime}(t)-z_{1}^{\prime}(t)\right)}{z_{2}(t)-z_{1}(t)} z+\frac{z_{1}(t) z_{2}^{\prime}(t)-z_{2}(t) z_{1}^{\prime}(t)}{z_{2}(t)-z_{1}(t)}=0 .
$$

After some computations we get that

$$
F=\frac{z-z_{1}(t)}{z_{2}(t)-z_{1}(t)}:=\Lambda(t)\left(z-z_{1}(t)\right)=
$$

is an invariant of the differential equation (5).

By Theorem 3 the first order Riccati differential equation having the three solutions $z_{1}(t), z_{2}(t)$ and $z_{3}(t)$ satisfying (8) for $n=2$ and $m=0$ can be written as (25). By considering that Ricatti equation under the change $w=1 /\left(z-z_{3}\right)$ can be transformed to the linear differential equation (26), which has the invariant

$$
F=\frac{w-w_{1}(t)}{w_{2}(t)-w_{1}(t)},
$$


(see formula (31)). Clearly this differential equation has the solution $w_{1}=1 /\left(z_{1}-z_{3}\right), w_{2}=$ $1 /\left(z_{2}-z_{3}\right)$, then in view of formula (31) we have that this linear equation has the invariant

$$
F=-\frac{w-w_{3}}{w_{1}-w_{2}}=-\frac{1 /\left(z-z_{3}\right)-1 /\left(z_{1}-z_{3}\right)}{1 /\left(z_{1}-z_{3}\right)-1 /\left(z_{2}-z_{3}\right)}=\frac{\left(z-z_{1}\right)\left(z_{2}-z_{3}\right)}{\left(z-z_{3}\right)\left(z_{2}-z_{3}\right)}:=\Lambda(t) \frac{\left(z-z_{1}\right)}{\left(z-z_{3}\right)} .
$$

The invariant for the linear differential equations is trivial and for the Riccati case corresponds with the well known fact that the cross-ratio of four linearly independent solutions is a constant (see for instance [2]). These results are known but the interest of the proofs which we propose consists in showing that these proofs can obtained from the inverse approach: we have the explicit expression of these differential equations for the given solutions.

\section{Abel Differential equations having an invariant}

The main objective of this subsection is to study the problem of existence of the invariant (3) for the Abel differential equations .

From Theorem 3 the Abel equation of the first kind (13) having four solutions $z_{1}, z_{2}, z_{3}, z_{4}$ satisfying (8) for $n=3$ and $m=0$ can be written as (27) Clearly that in this case

$$
\begin{aligned}
p(t)= & -\frac{\Delta_{1}(\omega)}{\Delta(\omega)}=\frac{z_{1}^{\prime}}{\left(z_{1}-z_{2}\right)\left(z_{1}-z_{3}\right)\left(z_{1}-z_{4}\right)}+\frac{z_{2}^{\prime}}{\left(z_{2}-z_{1}\right)\left(z_{2}-z_{3}\right)\left(z_{2}-z_{4}\right)} \\
& +\frac{z_{3}^{\prime}}{\left(z_{3}-z_{2}\right)\left(z_{3}-z_{1}\right)\left(z_{3}-z_{4}\right)}+\frac{z_{4}^{\prime}}{\left(z_{4}-z_{2}\right)\left(z_{4}-z_{3}\right)\left(z_{4}-z_{1}\right)}, \\
q(t)= & \frac{\Delta_{2}(\omega)}{\Delta(\omega)}, \quad r(t)=-\frac{\Delta_{3}(\omega)}{\Delta(\omega)}, \quad s(t)=\frac{\Delta_{4}(\omega)}{\Delta(\omega)} .
\end{aligned}
$$

As we prove in section 3, the Abel equation of first kind (27) under the change $z=z_{4}(t)+\frac{1}{w}$, and introducing the respectively notations, becomes into the Abel equation of a second kind (28) or equivalent (14).

Proof of Proposition 5. We consider

$$
\frac{d F}{d t}=\left(-\sum_{j=1}^{3} m_{j} \Phi(t)+\sum_{j=1}^{3} m_{j} \frac{w^{\prime}-w_{j}^{\prime}(t)}{w-w_{j}(t)}\right) F .
$$

Then in view of (14) and by considering that $w_{j}(t)$ is a solution of (14), i.e.

$$
w_{j} w_{j}^{\prime}+\Psi(t) w_{j}=\Phi w_{j}^{2}+\Upsilon(t)
$$

then (32) becomes

$$
\begin{aligned}
& -\sum_{j=1}^{3} m_{j} \Phi(t)+\sum_{j=1}^{3} m_{j} \frac{w^{\prime}-w_{j}^{\prime}(t)}{w-w_{j}(t)} \\
= & -\sum_{j=1}^{3} m_{j} \Phi(t)+\sum_{j=1}^{3} m_{j} \frac{\Phi(t) w-\Psi(t)+\frac{\Upsilon(t)}{w}-\Phi(t) w_{j}+\Psi(t)-\frac{\Upsilon(t)}{w_{j}}}{w-w_{j}(t)} \\
= & -\frac{\Upsilon(t)}{z} \sum_{j=1}^{3} \frac{m_{j}}{w_{j}}=0 .
\end{aligned}
$$


Hence (32) can be written as $\frac{d F}{d t}=\left(-\frac{\Upsilon(t)}{u} \sum_{j=1}^{3} \frac{m_{j}}{w_{j}}\right) F$. Since $\Upsilon(t) \neq 0$, from this last equality we have that $d F / d t=0$ if and only if $\sum_{j=1}^{3} m_{j} / w_{j}(t)=0$. The proof of representation (15) it is easy to obtain. Hence this completes the proof of the proposition.

Proof of Corollary 6. It follows from Proposition 9 by considering that $w=\frac{1}{z-z_{4}}$, and $w_{j}=\frac{1}{z_{j}-z_{4}}$, for $j=1,2,3$, consequently condition (16) becomes $\sum_{j=1}^{3} \frac{m_{j}}{w_{j}}=\sum_{j=1}^{3} m_{j}\left(z_{j}-\right.$ $\left.\left.z_{4}\right)\right)=0$.

Example 4. We illustrated Corollary 6 for the case when $z_{j}$ for $j=1,2,3$ are constants. After some computations we can prove that differential equation (27) becomes

$$
\dot{z}=\frac{\left(z-z_{1}\right)\left(z-z_{2}\right)\left(z-z_{3}\right) \dot{z}_{4}(t)}{\left(z_{4}(t)-z_{1}\right)\left(z_{4}(t)-z_{2}\right)\left(z_{4}(t)-z_{3}\right)}
$$

The invariant $F$ in this case is (see invariant (11))

$$
f F=\left(\frac{z-z_{1}}{z_{4}(t)-z_{1}}\right)^{m_{1}}\left(\frac{z-z_{2}}{z_{4}(t)-z_{2}}\right)^{m_{2}}\left(\frac{z-z_{3}}{z_{4}(t)-z_{3}}\right)^{m_{3}}
$$

where

$$
m_{1}=\frac{1}{\left(z_{1}-z_{2}\right)\left(z_{1}-z_{3}\right)}, \quad m_{2}=\frac{1}{\left(z_{2}-z_{1}\right)\left(z_{2}-z_{3}\right)}, \quad m_{3}=\frac{1}{\left(z_{3}-z_{1}\right)\left(z_{3}-z_{2}\right)},
$$

clearly that $m_{1}+m_{2}+m_{3}=0$ and $m_{1} z_{1}+m_{2} z_{2}+m_{3} z_{3}=0$.

Clearly after the change of variables $w \longmapsto w e e^{\int \Phi d t}$ equation (14) becomes

$$
w\left(w^{\prime}+\Psi(t)\right)=\Upsilon(t) .
$$

Proposition 9. Let the sets of functions

$$
\omega=\left\{w_{1}, w_{2}, w_{3}\right\}, \quad \omega_{\kappa}=\left\{w_{1}^{(\kappa)}, w_{2}^{(\kappa)}, w_{3}^{(\kappa)}\right\}, \quad \text { for } 1 \leq \kappa \leq \varsigma,
$$

be equivalent sets of solutions of the Abel differential equation of second kind (13). Denoting by $\Omega=\left\{w_{1}, w_{2}, w_{3}, w_{4}=w_{1}^{(1)}, w_{5}=w_{2}^{(1)}, w_{6}=w_{3}^{(\varsigma)}, \ldots\right\}$ the set of all equivalent solutions where $\kappa \in \mathbb{N}$ and $\mu=3(\varsigma+1)$. Then equation (14) has the invariant

$$
F=e^{-\sum_{j=1}^{\mu} m_{j} \int \Phi(t) d t} \prod_{j=1,}^{\mu}\left(w-w_{j}\right)^{m_{j}}, \text { with } w_{j} \in \Omega,
$$

if and only if

$$
\sum_{j=1}^{\mu} \frac{m_{j}}{w_{j}}=0
$$

Here the $m_{j}$ are constants not all zero. Moreover, under the changes

$$
w=W e e^{\int \Phi d t}, \quad w_{j}=W_{j} e^{\int \Phi d t},
$$


the invariant $F$ becomes

$$
F=\prod_{j=1}^{\mu}\left(W-W_{j}(t)\right)^{m_{j}}
$$

Corollary 10. Let the sets of functions

$$
\omega_{1}=\left\{z_{1}, z_{2}, z_{3}, z_{4}\right\}, \quad \omega_{\kappa}=\left(z_{1}^{(\kappa)}, z_{2}^{(\kappa)}, z_{3}^{(\kappa)}, z_{4}^{(\kappa)}\right\}, \quad \text { for } \quad 1 \leq \kappa \leq \varsigma,
$$

be equivalent sets of solutions of the Abel differential equation of first kind (13). Denoting by $\Omega=\left\{z_{1}, z_{2}, z_{3}, z_{4}, z_{5}=z_{1}^{(1)}, z_{6}=z_{2}^{(1)}, z_{7}=z_{3}^{(\varsigma)}, z_{8}=z_{4}^{(\varsigma)}, \ldots\right\}$ the set of all equivalent solutions. Then there exists the invariant

$$
F=\Lambda(t) \prod_{k=1, k \neq 4}^{\mu}\left(\frac{z-z_{j}}{z-z_{4}}\right)^{m_{j}}, \quad \text { with } \quad z_{j} \in \Omega,
$$

if and only if

$$
\sum_{j=1, j \neq 4}^{\mu} m_{j}\left(z_{j}-z_{4}\right)=0
$$

Moreover if $\sum_{j=1}^{\mu} m_{j}=0$ then $\sum_{j=1}^{\mu} m_{j} z_{j}=0$ and the invariant $F$ becomes

$$
F=\prod_{k=1, k \neq 4}^{\mu}\left(\frac{z-z_{j}}{z_{j}-z_{4}}\right)^{m_{j}}=\Lambda(t) \prod_{k=1, k \neq 4}^{\mu}\left(z-z_{j}\right)^{m_{j}}
$$

Proof. It is analogous to the proof of Proposition 9.

There are a lot of results on the existence of invariants $F$ for the equation (17), or for its canonical form (see for instance [2]).

Introducing the new independent variable $x=-\int \Psi(t) d t$ we get the canonical form of the Abel equation of the second kind becomes

$$
u\left(\frac{d u}{d x}-1\right)=R(x) \quad \text { with } \quad R(x)=\frac{-\Upsilon}{\Psi} .
$$

Clearly that this differential equation with two solutions $\omega=\left\{u_{1}, u_{2}\right\}$ where $u_{1}=u_{1}(x)$ and $u_{2}=u_{2}(x)$, can be written as

$$
u u^{\prime}+\frac{\Delta_{1}(\omega)}{\Delta(\omega)} u+\frac{\Delta_{2}(\omega)}{\Delta(\omega)}=0
$$

where $^{\prime}:=\frac{d}{d x}$, and $\frac{\Delta_{1}\left(\omega_{1}\right)}{\Delta\left(\omega_{1}\right)}=-1, \quad \frac{\Delta_{2}\left(\omega_{1}\right)}{\Delta\left(\omega_{1}\right)}=R(x)$

Proposition 11. Let the sets of functions

$$
\omega_{1}=\left\{u_{1}, u_{2}\right\}, \quad \omega_{\kappa}=\left\{u_{1}^{(\kappa)}, u_{2}^{(\kappa)}\right\}, \quad \text { for } 1 \leq \kappa \leq \varsigma,
$$

be equivalent sets of solutions of the Abel differential equation of second kind (13). Denoting by $\Omega=\left\{u_{1}, u_{2}, u_{3}=w_{1}^{(1)}, u_{4}=w_{2}^{(1)}, \ldots\right\}$ the set of all equivalent solutions of the Abel differential equation of second kind (34). Then there exists the invariant

$$
F=\prod_{k=1, k}^{\mu}\left(u-u_{j}\right)^{m_{j}}, \quad \text { with } \quad u_{j} \in \Omega
$$


if and only if

$$
\sum_{j=1,}^{\mu} \frac{m_{j}}{u_{j}}=0
$$

Proof. It is analogous to the proof of Proposition 9 with $\Phi=0$ and $\Psi=-1$.

Example 5. We study equation (34) in the particular case

$$
u\left(\frac{d u}{d x}-1\right)=\frac{3 x}{16}-\frac{A}{\sqrt[3]{x}}-\frac{B}{\sqrt[3]{x^{5}}} .
$$

In [2] the authors showed that the set of function $\omega_{1}=\left\{u_{1}, u_{2}\right\}$ where

$$
\begin{aligned}
& u_{1}=\frac{3}{4} x+\sqrt{3 A+\frac{3}{2} \sqrt{-3 B}} \sqrt[3]{x}+\frac{\sqrt{-3 B}}{\sqrt[3]{x}}, \\
& u_{2}=\frac{3}{4} x-\sqrt{3 A+\frac{3}{2} \sqrt{-3 B}} \sqrt[3]{x}+\frac{\sqrt{-3 B}}{\sqrt[3]{x}},
\end{aligned}
$$

are solutions of equation (36). The following set of functions $\omega_{2}=\left\{u_{3}, u_{4}\right\}$

$$
\begin{aligned}
& u_{3}=\frac{3}{4} x+\sqrt{3 A-\frac{3}{2} \sqrt{-3 B}} \sqrt[3]{x}-\frac{\sqrt{-3 B}}{\sqrt[3]{x}}, \\
& u_{4}=\frac{3}{4} x-\sqrt{3 A-\frac{3}{2} \sqrt{-3 B}} \sqrt[3]{x}-\frac{\sqrt{-3 B}}{\sqrt[3]{x}},
\end{aligned}
$$

is equivalent to (37). Indeed it is easy to show that

$$
\begin{aligned}
& \frac{\Delta_{1}\left(\omega_{1}\right)}{\Delta\left(\omega_{1}\right)}=\frac{\Delta_{1}\left(\omega_{2}\right)}{\Delta\left(\omega_{2}\right)}=-1, \\
& \frac{\Delta_{2}\left(\omega_{1}\right)}{\Delta\left(\omega_{1}\right)}=\frac{\Delta_{2}\left(\omega_{2}\right)}{\Delta\left(\omega_{2}\right)}=\frac{3 x}{16}-\frac{A}{\sqrt[3]{x}}-\frac{B}{\sqrt[3]{x^{5}}}
\end{aligned}
$$

By considering that $m_{1} / u_{1}+m_{2} / u_{2}+m_{3} / u_{3}+m_{4} / u_{4}=0$, where

$$
\begin{aligned}
& m_{1}=\sqrt{12 A-6 \sqrt{-3 B}}, \quad m_{2}=-\sqrt{12 A-6 \sqrt{-3 B}}, \\
& m_{3}=-\sqrt{12 A+6 \sqrt{-3 B}}, \quad m_{4}=\sqrt{12 A+6 \sqrt{-3 B}} .
\end{aligned}
$$

Then in view of Proposition 11 we obtain that equation (36) has the invariant

$$
\begin{aligned}
F & =\left(u-u_{1}\right)^{m_{1}}\left(u-u_{2}\right)^{m_{2}}\left(u-u_{3}\right)^{m_{3}}\left(\left(u-u_{4}\right)^{m_{4}},\right. \\
& =\left(\frac{u-u_{1}}{u-u_{2}}\right)^{m_{1}}\left(\frac{u-u_{4}}{u-u_{3}}\right)^{m_{4}} .
\end{aligned}
$$

According to the result given by Alexeeva, Zaitsev and Zhvets [2], any Abel equation of the second kind in the canonical form (33) has the invariant

$$
F=\prod_{j=1,}^{m}\left(u-u_{j}(s)\right)^{m_{j}}
$$

where $u_{j}(s)$ is a particular solution of (33) and $m_{j}$ is a convenient constant for $j=1, \ldots, m$ if the following relation holds

$$
\sum_{j=1}^{m}\left(\left(m_{j}\left(\frac{d u}{d s}-\frac{d u_{j}(s)}{d s}\right) \prod_{k=1, . ., n}^{k \neq j}\left(u-u_{k}(s)\right)\right)=\sum_{j=1}^{m}\left(\frac{d u}{d s} \Phi_{j}(s)+\Psi_{j}(s) u^{j}\right),\right.
$$

where $\Phi_{\nu}(s)=-\Psi_{\nu}(s) \quad \Psi_{\nu-1}(s)=-R(s) \Phi_{\nu}(s)$, and equate the other $\Phi_{k}$ and $\Psi_{k}$ with zero. 
Selecting different values $\nu=1, \ldots, n-1$, we obtain $n-1$ systems of different-algebraic equations; only one of the systems, corresponding to $m_{k} \neq 0$ for all $k=1, \ldots, n$ and $u_{i} \neq u_{j}$ for $i \neq j$, leads to a non degenerate solution of the form (38). Clearly condition (39) is complicated to compute unlike conditions (17). In the monograph [2] there is an example of solutions of (33) obtained from (39) for a convenient $R(x)$. All these solutions satisfy (35) for a convenient constants $m_{j}$. The examples given in [2] are the following and as we show all of them satisfy the condition (35).

Example 6. For $n=2$ differential equation (33) with $R(x)=A x+b$ where a and $B$ are constants has the following solutions

$$
u_{1}=\frac{1+\sqrt{1+4 A}}{2 A}(A x+b), \quad u_{2}==-\frac{1+\sqrt{1+4 A}}{2 A+1+\sqrt{1+4 A}}(A x+b),
$$

and $m_{1}=2 A+1+\sqrt{1+4 A}, m_{2}=2 A$. Clearly that $m_{1} / u_{1}+m_{2} / u_{2}=0$.

For $n=3$ and for $R(x)=-2 / 9 t x+A+B / \sqrt{x}$ the obtained solutions in [2] are

$$
\begin{aligned}
u_{j} & =\frac{2}{3}\left(x+\lambda_{j} \sqrt{x}\right)+\frac{3 B}{\lambda_{j}} \\
m_{j} & =\frac{2 A}{3\left(2 \lambda_{j}^{2}-3 A\right)} \text { for } j=1,2,3,
\end{aligned}
$$

where $\lambda$ is a root of $\lambda^{3}-9 / 2 A \lambda-9 / 2 B=0$. It is easy to show that $m_{1} / u_{1}+m_{2} / u_{2}+$ $m_{3} / u_{3}=0$. We can prove that $\Omega=\left\{u_{1}, u_{2}, u_{3}\right\}$ is a set of equivalent solutions. Indeed if $\omega_{1}=\left\{u_{1}, u_{2}\right\}, \omega_{2}=\left\{u_{1}, u_{3}\right\}$ and $\omega_{3}=\left\{u_{2}, u_{3}\right\}$ then

$$
\begin{aligned}
& \frac{\Delta_{1}\left(\omega_{1}\right)}{\Delta\left(\omega_{1}\right)}=\frac{\Delta_{1}\left(\omega_{2}\right)}{\Delta\left(\omega_{2}\right)}=\frac{\Delta_{1}\left(\omega_{3}\right)}{\Delta\left(\omega_{3}\right)}=-1, \\
& \frac{\Delta_{2}\left(\omega_{1}\right)}{\Delta\left(\omega_{1}\right)}=\frac{\Delta_{2}\left(\omega_{2}\right)}{\Delta\left(\omega_{2}\right)}=\frac{\Delta_{1}\left(\omega_{3}\right)}{\Delta\left(\omega_{3}\right)}==-2 / 9 x+A+B / \sqrt{x} .
\end{aligned}
$$

For $m=4$ and $\frac{3 x}{16}-\frac{A}{\sqrt[3]{x}}-\frac{B}{\sqrt[3]{x^{5}}}$ the authors proposed the solutions given in example 5 with wrong constants $m_{1}=-m_{2}=-2 A+\sqrt{-3 B}$, and $m_{3}=-m_{4}=\sqrt{4 A^{2}+3 B}$, because $m_{1} / u_{1}+m_{2} / u_{2}+m_{3} / u_{3}+m_{4} / u_{4} \neq 0$.

5.1. Julia invariant. Assume that the functions $u_{1}(s), \ldots, u_{\mu}(s)$ satisfy the equation

$$
u^{\mu}+a_{1}(s) u^{\mu-1}+\ldots+a_{\mu-2}(s) u^{2}+a_{\mu}(s)=0,
$$

and are solutions of the canonic Abel second kind differential equation (33), then there exists an invariant

$$
F=\prod_{j=1}^{\mu}\left(u-u_{j}(s)\right)
$$

Indeed, under the given assumption the functions $u_{1}(s), \ldots, u_{m}(s)$ satisfy the relation

$$
\prod_{j=2}^{\mu} u_{j}(s)+\prod_{j=1, j \neq 2}^{\mu} u_{j}(s)+\ldots+\prod_{j=1}^{\mu-1} u_{j}(s)=0
$$

or what is equivalent $\sum_{j=1}^{\mu} \frac{1}{u_{j}(s)}=0$, consequently, in view of Proposition 11 with $m_{j}=1$ for $j=1, \ldots, \mu$ there exists the invariant $F$. 
In particular if $\mu=2$ we have that if $\frac{1}{u_{1}}+\frac{1}{u_{2}}=0$ or, what is equivalent $u_{1}+u_{2}=0$, then the invariant becomes

$$
F=\left(u-u_{1}\right)\left(u+u_{1}\right)
$$

The French mathematician Gaston Julia proved 1933 in [5] (6, p. 82f) that the equation

$$
(D y+E) \frac{d y}{d t}+A y^{2}+B y+C=0,
$$

for $A, B, C, D$ and $E$ functions of $t$, has an implicit solution if the condition

$$
L:=E\left(2 A-D^{\prime}\right)-D\left(B-E^{\prime}\right)=0,
$$

with $D \neq 0$ is satisfied. Then the invariant is

$$
F=e^{\int \frac{2 A}{D} d t}\left(\frac{y^{2}}{2}+\frac{E}{D} y\right)+\int \frac{C}{D} e^{\int \frac{2 A}{D} d t}=\text { const }
$$

Doing two changes of variables the previous differential equation yields the general Abel equation of first kind. First we do the change of variables $y=w(t)-\frac{E}{D}$ and we get

$$
w w^{\prime}+\frac{A}{D} w^{2}+\frac{L}{D^{2}} w+\frac{E^{2} A-E D B+C D^{2}}{D^{3}}=0 .
$$

The invariant under the given change becomes

$$
F=e^{\int \frac{2 A}{D} d t}\left(w^{2}-\frac{E^{2}}{D^{2}}\right)+\int \frac{C}{D} e^{\int \frac{2 A}{D} d t}
$$

By putting $L=0$ and after the change $w \longrightarrow w e^{\int \frac{A}{D} d t}$ we obtain that the differential equation (41) becomes

$$
w w^{\prime}+e \int \frac{-2 A}{D} d t\left(\frac{E^{2} A-E D B+C D^{2}}{D^{3}}\right)=0 .
$$

This last differential equation has the invariant $F=\left(u-u_{1}\right)\left(u+u_{1}\right)$, where

$$
u_{1}=-u_{2}=\sqrt{\frac{E^{2}}{D^{2}} e^{\int \frac{2 A}{D} d t}+\int \frac{C}{D} e^{\int \frac{2 A}{D} d t},}
$$

which corresponds to the invariant (40) with $\mu=2$.

Finally we observe that there exits a big numbers of papers devoted to study the existence of exactly solutions and applications of the Abel equation (see for instance [1, 3, 7, 8, 9, 18]).

\section{Planar polynomial differential EQuations with Given SOlutions}

In this section we study the problem II.1. We determine the planar differential systems

$$
\dot{x}=P(x, y, t) \quad \dot{y}=Q(x, y, t),
$$

having the given set of solutions $\omega=\left\{z_{1}(t), \ldots, z_{k}(t)\right\}$ where $k \geq 1$.

After the change $z=x+i y$ and $\bar{z}=x-i y$ differential system (42) becomes

$$
\dot{z}=R(z, \bar{z}, t)
$$

where

$$
R=R(z, \bar{z}, t)=\left.(P+i Q)\right|_{x=(z+\bar{z}) / 2, y=(z-\bar{z}) /(2 i)} .
$$


First we determine the simplest case when $\omega=\left\{z_{1}(t)\right\}$.

Proposition 12. The most general planar analytic differential system with the given solution $z_{1}(t)$ can be written as

$$
\dot{z}=\dot{z}_{1}+\Phi(z, \bar{z}, t)
$$

or equivalently

$$
\left.\left.\dot{x}=\dot{x}_{1}(t)+\operatorname{Re} \Phi(z, \bar{z}, t)\right), \quad \dot{y}=\dot{y}_{1}(t)+\operatorname{Im} \Phi(z, \bar{z}, t)\right),
$$

where $z_{1}(t)=x_{1}(t)+i y_{1}(t)$ and $\Phi$ is any analytic function (Erugyn function) such that

$$
\Phi(z, \bar{z}, t))\left.\right|_{z=z_{1}(t), \bar{z}=\bar{z}_{1}(t)}=0 .
$$

Proof. Indeed $z=z_{1}(t)$ is a solution of (44) because (45) holds. Assume that differential system $\dot{z}=L(z, \bar{z}, t)$ is another differential equation having $z=z_{1}(t)$ as a solution, i.e. $L\left(z_{1}(t), z_{1}(t), t\right)=\dot{z}_{1}(t)$. taking $\Phi(z, \bar{z}, t)=L(z, \bar{z}, t)-\dot{z}_{1}(t)$ we get that this differential equation is a particular case of (44).

Proposition 13. The most general planar polynomial differential system of degree $m$ with coefficients functions depending on $t$ and with the given solution $z_{1}(t)$, can be written as (44) with either

$$
\Phi=\alpha_{1}(t) H_{11}+\alpha_{2}(t) H_{12}+\alpha_{3}(t) H_{21}+\alpha_{4}(t) H_{22}=<\tilde{\xi}(t), M(t) \xi^{T}>,
$$

where $\alpha_{j}(t)$ are arbitrary functions, $H_{j k}$ is a component of the matrix

$$
H=G(t, z, \bar{z}) G\left(t, z_{1}(t), \bar{z}_{1}(t)\right)-G\left(t, z_{1}(t), \bar{z}_{1}(t)\right) G(t, z, \bar{z}):=\left[G(t, z, \bar{z}), G\left(t, z_{1}(t), \bar{z}_{1}(t)\right)\right],
$$

with the matrix

$$
G(t, z, \bar{z})=\left(\begin{array}{cc}
1 & 1 \\
p_{1}(t, z, \bar{z}) & p_{2}(t, z, \bar{z})
\end{array}\right)
$$

and

$$
\begin{aligned}
p_{1}(t, z, \bar{z}) & =\sum_{n+j=0}^{m} a_{n j}(t) z^{n} \bar{z}^{j}, \quad p_{2}(t, z, \bar{z})=\sum_{n+j=0}^{m} b_{n j}(t) z^{n} \bar{z}^{j}, \\
\xi & =\left(1, z, \bar{z}, z^{2}, z \bar{z}^{2}, \bar{z}^{2}, \ldots, z^{m}, \ldots, \bar{z}^{m}\right), \quad \tilde{\xi}(t)=\left.\xi\right|_{z=z_{1}(t), \bar{z}=\bar{z}_{1}(t)},
\end{aligned}
$$

and $M(t)$ is a convenient $(m+1)(m+2) / 2 \times(m+1)(m+2) / 2$ antisymmetric matrix with components functions of $t$. In particular, the most general differential equation with a given singular point $z_{1}(t)=\alpha+i \beta \in \mathbb{C}$ can be written as $(44)$ with $\dot{z}_{1}(t)=0$.

Proof. The proof of this statement require a huge number of computations. We prove this proposition only for linear and quadratic differential equations.

First we study the linear case, when $p_{1}(t, z, \bar{z})=z, p_{2}(t, z, \bar{z})=\bar{z}$ Hence

$$
\begin{aligned}
H & =\left(\begin{array}{ll}
1 & 1 \\
z & \bar{z}
\end{array}\right)\left(\begin{array}{cc}
1 & 1 \\
z_{1}(t) & \bar{z}(t)
\end{array}\right)-\left(\begin{array}{cc}
1 & 1 \\
z_{1}(t) & \bar{z}(t)
\end{array}\right)\left(\begin{array}{cc}
1 & 1 \\
z & \bar{z}
\end{array}\right) \\
& =\left(\begin{array}{cc}
z_{1}(t)-z & \bar{z}_{1}(t)-\bar{z} \\
\bar{z} z_{1}(t)-\bar{z}_{1} z+z-z_{1}(t) & z-z_{1}(t)
\end{array}\right)
\end{aligned}
$$

Consequently

$$
\begin{aligned}
\Phi & =\tilde{\alpha}_{1}(t)\left(z-z_{1}(t)\right)-\alpha_{2}(t)\left(\bar{z}-\bar{z}_{1}(t)\right)+\alpha_{3}(t)\left(\bar{z} z_{1}(t)-z \bar{z}_{1}(t)\right) \\
& =\left(\begin{array}{cccc}
1, & z_{1}(t), & \bar{z}_{1}(t)
\end{array}\right)\left(\begin{array}{ccc}
0 & \tilde{\alpha}_{1}(t) & -\alpha_{2}(t) \\
-\tilde{\alpha}_{1}(t) & 0 & \alpha_{3}(t) \\
\alpha_{2}(t) & -\alpha_{3}(t) & 0
\end{array}\right)\left(\begin{array}{c}
1 \\
z \\
\bar{z}
\end{array}\right),
\end{aligned}
$$


where $\tilde{\alpha}_{1}(t)=-\alpha_{1}(t)+\alpha_{3}(t)+\alpha_{4}(t)$. Thus from (44) we obtain that the most general planar linear differential system of first order with the given solution $z_{1}(t)$ is

$$
\dot{z}=\dot{z}_{1}+\tilde{\alpha}_{1}(t)\left(z-z_{1}(t)\right)-\alpha_{2}(t)\left(\bar{z}-\bar{z}_{1}(t)\right)+\alpha_{3}(t)\left(\bar{z} z_{1}(t)-z \bar{z}_{1}(t)\right),
$$

where $\tilde{\alpha}_{1}(t), \alpha_{2}(t)$ and $\alpha_{3}(t)$ are arbitrary functions. So the proposition is proved for the linear differential equations of first order with the given solution $z_{1}(t)$.

For the quadratic differential systems we have that

$$
\begin{aligned}
\tilde{\xi}(t) M(t) \xi(t)= & -\left(a_{46} z_{1}^{2}+a_{56} z_{1} w_{1}+a_{26} z_{1}+a_{36} \bar{z}_{1}+a_{16}\right) \bar{z}^{2} \\
& \left(a_{56} w_{1}^{2}-a_{45} z_{1}^{2}-a_{25} z_{1}-a_{35} \bar{z}_{1}-a_{15}\right) z \bar{z} \\
& +\left(a_{45} z_{1} \bar{z}_{1}+a_{46} \bar{z}_{1}^{2}-a_{24} z_{1}-a_{34} \bar{z}_{1}-a_{14}\right) z^{2} \\
& \left(a_{34} z_{1}^{2}+a_{35} z_{1} \bar{z}_{1}+a_{36} \bar{z}_{1}^{2}-a_{23} z_{1}-a_{13}\right) \bar{z} \\
& +\left(a_{24} z_{1}^{2}+a_{25} z_{1} \bar{z}_{1}+a_{26} \bar{z}_{1}^{2}+a_{23} z_{1}-a_{12}\right) z \\
& a_{14} z_{1}^{2}+a_{15} z_{1} \bar{z}_{1}+a_{16} z_{1}^{2}+a_{12} z_{1}+a_{13} \bar{z}_{1}
\end{aligned}
$$

where $M_{j k}=a_{j k}(t)$. From the equation $\Phi=\sigma_{1} z^{2}+\sigma_{2} \bar{z}^{2}+\sigma_{3} z \bar{z}+\sigma_{4} z+\sigma_{5} \bar{z}=\tilde{\xi}(t) M(t) \xi(t)$ we get that

$$
\begin{aligned}
\sigma_{1} & =a_{16}+a_{46} z_{1}^{2}+a_{56} z_{1} w_{1}+a_{26} z_{1}+a_{36} \bar{z}_{1}, \\
\sigma_{2} & =-a_{15}+a_{56} w_{1}^{2}-a_{45} z_{1}^{2}-a_{25} z_{1}-a_{35} \bar{z}_{1}, \\
\sigma_{3} & =-a_{14}+a_{45} z_{1} \bar{z}_{1}+a_{46} \bar{z}_{1}^{2}-a_{24} z_{1}-a_{34} \bar{z}_{1}, \\
\sigma_{4} & =-a_{13}+a_{34} z_{1}^{2}+a_{35} z_{1} \bar{z}_{1}+a_{36} \bar{z}_{1}^{2}, \\
\sigma_{5} & =-a_{12}+a_{24} z_{1}^{2}+a_{25} z_{1} \bar{z}_{1}+a_{26} \bar{z}_{1}^{2}+a_{23} z_{1}, \\
\left.\Phi\right|_{z=z_{1}, \bar{z}=\bar{z}_{1}} & =\sigma_{1} z_{1}^{2}+\sigma_{2} \bar{z}_{1}^{2}+\sigma_{3} z_{1} \bar{z}_{1}+\sigma_{4} z_{1}+\sigma_{5} \bar{z}_{1}=0 .
\end{aligned}
$$

This completes the proof of the proposition for the quadratic polynomial differential systems with a given solution $z_{1}(t)$.

In similar way we could obtain the result of Proposition 13 for polynomial differential equations of degree $m>2$ with a given solution $z_{1}(t)$, but the computations increases strongly.

Proposition 14. The most general planar polynomial differential system of degree $m$ with the given solutions $\omega=\left\{z_{1}(t), 0\right\}$, can be written as

$$
\dot{z}=\frac{\dot{z}_{1}(t)}{z_{1}(t)} z+\Phi(z, \bar{z}, t),
$$

where

$$
\Phi(z, \bar{z}, t)=<\tilde{\xi}(t), M(t) \xi^{T}>
$$

and $\xi=\left(z, \bar{z}, z^{2}, z \bar{z}^{2}, \bar{z}^{2}, \ldots, z^{m}, \ldots, \bar{z}^{m}\right), \tilde{\xi}(t)=\left.\xi\right|_{z=z_{1}(t), \bar{z}=\bar{z}_{1}(t)}$ and $M=M(t)$ is a $m(m+$ $3) / 2 \times m(m+3) / 2$ antisymmetric matrix with components arbitrary functions of $t$. In particular, the most general differential equation with a given singular points $z_{1}(t)=\alpha+i \beta \in$ $\mathbb{C}$ and $z_{2}=0$ can be written as $(46)$ with $\dot{z}_{1}(t)=0$.

Proof. It is analogous to the proof of Proposition 13. 


\subsection{Construction the quadratic differential system with a given set of solutions.}

Proposition 15. Let $\omega=\left\{z_{1}(t), z_{2}(t), z_{3}(t), z_{4}(t), z_{5}(t), z_{6}(t)\right\}$ be a set of functions such that

$$
\Delta(\omega):=\left|\begin{array}{cccccc}
1 & 1 & 1 & 1 & 1 & 1 \\
z_{1} & z_{2} & z_{3} & z_{4} & z_{5} & z_{6} \\
\bar{z}_{1} & \bar{z}_{2} & \bar{z}_{3} & \bar{z}_{4} & \bar{z}_{5} & \bar{z}_{6} \\
z_{1}^{2} & z_{2}^{2} & z_{3}^{2} & z_{4}^{2} & z_{5}^{2} & z_{6}^{2} \\
\bar{z}_{1}^{2} & \bar{z}_{2}^{2} & \bar{z}_{3}^{2} & \bar{z}_{4}^{2} & \bar{z}_{5}^{2} & \bar{z}_{6}^{2} \\
z_{1} \bar{z}_{1} & z_{2} \bar{z}_{2} & z_{3} \bar{z}_{3} & z_{4} \bar{z}_{4} & z_{5} \bar{z}_{5} & z_{6} \bar{z}_{6}
\end{array}\right| \neq 0
$$

where $\sum_{j=1}^{\kappa}\left|\dot{z}_{j}\right|^{2} \neq 0$. Then the most general quadratic differential equations of the first order

$$
\dot{z}=a_{0}(t)+a_{1}(t) z+a_{2}(t) \bar{z}+a_{3}(t) z^{2}+a_{4}(t) z \bar{z}+a_{5}(t) \bar{z}^{2},
$$

for which $\omega$ is a given set of solutions can be rewritten as

$$
\left|\begin{array}{ccccccc}
1 & 1 & 1 & 1 & 1 & 1 & 1 \\
z & z_{1} & z_{2} & z_{3} & z_{4} & z_{5} & z_{6} \\
\bar{z} & \bar{z}_{1} & \bar{z}_{2} & \bar{z}_{3} & \bar{z}_{4} & \bar{z}_{5} & \bar{z}_{6} \\
z^{2} & z_{1}^{2} & z_{2}^{2} & z_{3}^{2} & z_{5}^{4} & z_{5}^{2} & z_{6}^{2} \\
\bar{z}^{2} & \bar{z}_{1}^{2} & \bar{z}_{2}^{2} & \bar{z}_{3}^{2} & \bar{z}_{4}^{2} & \bar{z}_{5}^{2} & \bar{z}_{6}^{2} \\
z \bar{z} & z_{1} \bar{z}_{1} & z_{2} \bar{z}_{2} & z_{3} \bar{z}_{3} & z_{4} \bar{z}_{4} & z_{5} \bar{z}_{5} & z_{6} \bar{z}_{6} \\
\dot{z} & \dot{z}_{1} & \dot{z}_{2} & \dot{z}_{3} & \dot{z}_{4} & \dot{z}_{5} & \dot{z}_{6}
\end{array}\right|=0
$$

Moreover the quadratic vector field with the set of solutions $\omega=\left\{z_{1}, z_{2}, z_{3}, z_{4}, z_{5}, z_{6}\right\}$ where $z_{j}$ for $j=1,2,3,4,5$ are constants solutions such than (47) holds has the invariant

$$
\int\left(\frac{d z}{\Upsilon\left(z, z_{1}, z_{2}, z_{3}, z_{4}, z_{5}\right)}-\frac{d z_{6}}{\Upsilon\left(z_{6}, z_{1}, z_{2}, z_{3}, z_{4}, z_{5}\right)}\right)=C=\text { contstant }
$$

where

$$
\Upsilon\left(\xi, z_{1}, z_{2}, z_{3}, z_{4}, z_{5}\right)=\left|\begin{array}{cccccc}
1 & 1 & 1 & 1 & 1 & 1 \\
\xi & z_{1} & z_{2} & z_{3} & z_{4} & z_{5} \\
\bar{\xi} & \bar{z}_{1} & \bar{z}_{2} & \bar{z}_{3} & \bar{z}_{4} & \bar{z}_{5} \\
\xi^{2} & z_{1}^{2} & z_{2}^{2} & z_{3}^{2} & z_{5}^{4} & z_{5}^{2} \\
\bar{\xi}^{2} & \bar{z}_{1}^{2} & \bar{z}_{2}^{2} & \bar{z}_{3}^{2} & \bar{z}_{4}^{2} & \bar{z}_{5}^{2} \\
\xi \bar{\xi} & z_{1} \bar{z}_{1} & z_{2} \bar{z}_{2} & z_{3} \bar{z}_{3} & z_{4} \bar{z}_{4} & z_{5} \bar{z}_{5}
\end{array}\right|
$$

Proof. By applying the property of the determinant we easily prove that $z=z_{j}(t)$ are solutions of (51). The reciprocity we prove as follows. From the equations

$$
\dot{z}_{j}=a_{0}(t)+a_{1}(t) z_{j}+a_{2}(t) \bar{z}_{j}+a_{3}(t) z_{j}^{2}+a_{4}(t) z_{j} \bar{z}_{j}+a_{5}(t) \bar{z}_{j}^{2}, \quad \text { for } \quad j=1, \ldots, 6,
$$


or equivalently

$$
\left(\begin{array}{ccccc}
1 & z_{1} & \bar{z}_{1} & \ldots & \bar{z}_{1}^{2} \\
1 & z_{2} & \bar{z}_{2} & \ldots & \bar{z}_{2}^{2} \\
1 & z_{3} & \bar{z}_{3} & \ldots & \bar{z}_{3}^{2} \\
\vdots & \vdots & \vdots & \ldots & \vdots \\
1 & z_{6} & \bar{z}_{6} & \ldots & \bar{z}_{6}^{2}
\end{array}\right)\left(\begin{array}{c}
a_{0} \\
a_{1} \\
a_{3} \\
\vdots \\
a_{6}
\end{array}\right)=\left(\begin{array}{c}
\dot{z}_{1} \\
\dot{z}_{2} \\
\dot{z}_{3} \\
\vdots \\
\dot{z}_{6}
\end{array}\right)
$$

we obtain that

$$
a_{0}=\frac{1}{\Delta(\omega)}\left|\begin{array}{cccccc}
z_{1} & z_{2} & z_{3} & z_{4} & z_{5} & z_{6} \\
\bar{z}_{1} & \bar{z}_{2} & \bar{z}_{3} & \bar{z}_{4} & \bar{z}_{5} & \bar{z}_{6} \\
z_{1}^{2} & z_{2}^{2} & z_{3}^{2} & z_{4}^{2} & z_{5}^{2} & z_{6}^{2} \\
\bar{z}_{1}^{2} & \bar{z}_{2}^{2} & \bar{z}_{3}^{2} & \bar{z}_{4}^{2} & \bar{z}_{5}^{2} & \bar{z}_{6}^{2} \\
z_{1} \bar{z}_{1} & z_{2} \bar{z}_{2} & z_{3} \bar{z}_{3} & z_{4} \bar{z}_{4} & z_{5} \bar{z}_{5} & z_{6} \bar{z}_{6} \\
\dot{z}_{1} & \dot{z}_{2} & \dot{z}_{3} & \dot{z}_{4} & \dot{z}_{5} & \dot{z}_{6}
\end{array}\right|:=\frac{\Delta_{0}(\omega)}{\Delta(\omega)},
$$

By inserting into (48) we get

$$
\dot{z}=\frac{\Delta_{0}(\omega)}{\Delta(\omega)}+\frac{\Delta_{1}(\omega)}{\Delta(\omega)} z+\frac{\Delta_{2}(\omega)}{\Delta(\omega)} \bar{z}+\frac{\Delta_{3}(\omega)}{\Delta(\omega)} z^{2}+\frac{\Delta_{4}(\omega)}{\Delta(\omega)} z \bar{z}+\frac{\Delta_{5}(\omega)}{\Delta(\omega)} \bar{z}^{2}
$$

after some computations we obtain (51). The proof of (50) it is easy to obtain. Indeed from the equation 


$$
\left|\begin{array}{ccccccc}
1 & 1 & 1 & 1 & 1 & 1 & 1 \\
z & z_{1} & z_{2} & z_{3} & z_{4} & z_{5} & z_{6} \\
\bar{z} & \bar{z}_{1} & \bar{z}_{2} & \bar{z}_{3} & \bar{z}_{4} & \bar{z}_{5} & \bar{z}_{6} \\
z^{2} & z_{1}^{2} & z_{2}^{2} & z_{3}^{2} & z_{5}^{4} & z_{5}^{2} & z_{6}^{2} \\
\bar{z}^{2} & \bar{z}_{1}^{2} & \bar{z}_{2}^{2} & \bar{z}_{3}^{2} & \bar{z}_{4}^{2} & \bar{z}_{5}^{2} & \bar{z}_{6}^{2} \\
z \bar{z} & z_{1} \bar{z}_{1} & z_{2} \bar{z}_{2} & z_{3} \bar{z}_{3} & z_{4} \bar{z}_{4} & z_{5} \bar{z}_{5} & z_{6} \bar{z}_{6} \\
\dot{z} & 0 & 0 & 0 & 0 & 0 & \dot{z}_{6}
\end{array}\right|=0 .
$$

and developing this determinant with respect to the last row we get (20). Clearly if $\sum_{j=1}^{\kappa}\left|\dot{z}_{j}\right|^{2}=0$, then differential equation (51) becomes $\dot{z}=0$.

Proof of Proposition 7. The problem on the construction the polynomial planar vector field (48) of degree $m$ from a given set of $(m+1)(m+2) / 2$ solutions is solved in Proposition 7. The proof of this result is analogous to the proof of Proposition 15.

The set of functions $\omega_{\kappa}=\left\{z_{1}^{(\kappa)}, \ldots, z_{\kappa}^{(\kappa)}\right)$ for $1 \leq \kappa \leq \mu$ we call equivalent to the set $\omega_{1}=\left\{\left(z_{1}(t), \ldots, z_{\kappa}(t)\right)\right\}$ if

$$
\frac{\Delta_{j}\left(\omega_{1}\right)}{\Delta\left(\omega_{1}\right)}=\frac{\Delta_{j}\left(\omega_{\kappa}\right)}{\Delta\left(\Upsilon_{\kappa}\right)}
$$

for $j=2, \ldots, \kappa$.

Another interesting problem will be to determine the set of solutions $\omega$ such that

$$
\frac{\Delta_{j}\left(\omega_{1}\right)}{\Delta\left(\omega_{1}\right)}=a_{j}=\text { constants }, \quad \text { for } \quad j=1, \ldots, 6 .
$$

Remark 16. By considering that $(m+1)(m+2) / 2<m^{2}$ for $m=2,3$ then the most general quadratic and cubic polynomial differential systems can be obtained from (19) taking $z_{j}=$ constants for $m=2,3$.

6.2. Complex polynomial differential equations of degree $m$ with $m+1$ real solutions. In this section we study the problem on the determination the complex polynomial differential system of degree $m$ of the form (21) with $m+1$ complex solution satisfying (22) and $m+1$ real solutions.

Proof of Proposition 8. Assume that (21) has $m+1$ solutions such that (22) holds. Then from (21) it follows that

$$
\dot{z}_{j}=\lambda_{0}+\lambda_{1} z_{j}+\ldots+\lambda_{m} z_{j}^{m}, \quad \text { for } \quad j=1, \ldots, m+1
$$


where $\lambda_{k}=\sum_{n=0}^{k} e^{i n \alpha} a_{k-n, n}(t),, \quad$ for $\quad k=0, \ldots, m$. By solving (52) with respect to $\lambda_{k}$ for $k=0, \ldots, m$, we get that

$$
\lambda_{0}=\frac{1}{\Delta(\omega)}\left|\begin{array}{ccccc}
\dot{z}_{1} & z_{1} & z_{1}^{2} & \ldots & z_{1}^{m} \\
\dot{z}_{2} & z_{2} & z_{2}^{2} & \ldots & z_{2}^{m} \\
\dot{z}_{3} & z_{3} & z_{3}^{2} & \ldots & z_{3}^{m} \\
\vdots & \vdots & \vdots & \ldots & \vdots \\
\dot{z}_{m} & z_{m+1} & z_{m+1}^{2} & \ldots & z_{m+1}^{m}
\end{array}\right|
$$

$$
\lambda_{m}=\frac{1}{\Delta(\omega)}\left|\begin{array}{ccccc}
1 & z_{1} & z_{1}^{2} & \ldots & \dot{z}_{1} \\
1 & z_{2} & z_{2}^{2} & \ldots & \dot{z}_{2} \\
1 & z_{3} & z_{3}^{2} & \ldots & \dot{z}_{3} \\
\vdots & \vdots & \vdots & \ldots & \vdots \\
1 & z_{m+1} & z_{m+1}^{2} & \ldots & \dot{z}_{m}
\end{array}\right|
$$

By considering that

$$
\begin{aligned}
& a_{m, 0}=\lambda_{m}-a_{m-1,1} e^{i \alpha}-a_{m-2,2} e^{2 i \alpha}-\ldots-a_{0, m} e^{i m \alpha}, \\
& a_{m-1,0}=\lambda_{m-1}-a_{m-2,1} e^{i \alpha}-\ldots-a_{0, m-1} e^{i(m-1) \alpha}, \\
& a_{00}=\lambda_{0} \text {, }
\end{aligned}
$$

and inserting them (21) we get

$$
\begin{aligned}
& \dot{z}-\sum_{j=0}^{m} \lambda_{j} z^{j}=a_{m-1,1}\left(\bar{z} z^{m-1}-e^{i \alpha} z^{m}\right) \\
& +a_{m-2,2}\left(\bar{z}^{2} z^{m-2}-e^{2 i \alpha} z^{m}\right)+\ldots+a_{0, m}\left(\bar{z}^{m}-e^{i m \alpha} z^{m}\right) \\
& +\ldots+a_{0,1}\left(\bar{z}-e^{i \alpha} z\right)=\left(\bar{z}-e^{i \alpha} z\right)\left(a_{m-1,1} z^{m-1}\right. \\
& +a_{m-2,2} z^{m-2}\left(\bar{z}+e^{i \alpha} z\right)+\ldots+a_{0, m}\left(\bar{z}^{m-1}+\bar{z}^{m-2} e^{i \alpha} z\right. \\
& \left.\left.+\ldots+e^{i(m-1) \alpha} z^{m-1}\right)+\ldots+a_{0,1}\right):=\left(\bar{z}-e^{i \alpha} z\right) P(z, z, t) .
\end{aligned}
$$

Hence in view of (53) we get that

$$
\dot{z}-\sum_{j=0}^{m} \lambda_{j} z^{j}=\frac{1}{\Delta(\omega)}\left|\begin{array}{cccccc}
1 & 1 & 1 & 1 & \ldots & 1 \\
z & z_{1} & z_{2} & z_{3} & \ldots & z_{m+1} \\
z^{2} & z_{1}^{2} & z_{2}^{2} & z_{3}^{2} & \ldots & z_{m+1}^{2} \\
\vdots & \vdots & \vdots & \vdots & \ldots & \vdots \\
z^{m} & z_{1}^{m} & z_{1}^{m} & z_{1}^{m} & \ldots & z_{m+1}^{m} \\
\dot{z} & \dot{z}_{1} & \dot{z}_{2} & \dot{z}_{3} & \ldots & \dot{z}_{m+1}
\end{array}\right| .
$$

Therefore we obtain the proof of the proposition.

Corollary 17. The most general complex polynomial differential equations of degree $m$ with $m+1$ real solutions $\omega=\left(z_{1}(t), \ldots, z_{m+1}(t)\right)$ such that the Vandermonde determinant (23) 
is non-zero can be written as

$$
\frac{1}{\Delta(\omega)}\left|\begin{array}{cccccc}
1 & 1 & 1 & 1 & \ldots & 1 \\
z & z_{1} & z_{2} & z_{3} & \ldots & z_{m+1} \\
z^{2} & z_{1}^{2} & z_{2}^{2} & z_{3}^{2} & \ldots & z_{m+1}^{2} \\
\vdots & \vdots & \vdots & \vdots & \ldots & \vdots \\
z^{m} & z_{1}^{m} & z_{1}^{m} & z_{1}^{m} & \ldots & z_{m+1}^{m} \\
\dot{z} & \dot{z}_{1} & \dot{z}_{2} & \dot{z}_{3} & \ldots & \dot{z}_{m+1}
\end{array}\right|=(\bar{z}-z) P(z, \bar{z}, t),
$$

where $P(z, \bar{z}, t)$ is a convenient polynomial of degree $m-1$.

Proof. It follows from Proposition 8 with $\alpha=0$.

Remark 18. From Proposition 17 it follows the next remarks.

(i) If a complex polynomial differential equation of degree $m$ has $m+1$ singular points such that $\bar{z}_{j}=e^{i \alpha} z_{j}$, for $j=1, \ldots, m+1$, then it has the singular straight line $\bar{z}-e^{i \alpha} z=0$, i.e. $(1-\cos \alpha) x+\sin \alpha y=0$. The proof of this result follows trivially from (24). Indeed, in this case differential equation (54) becomes

$$
\dot{z}=\left(\bar{z}-e^{i \alpha} z\right) \tilde{P}(z, z, t) .
$$

where $\tilde{P}(z, z, t)$ is a convenient polynomial of degree $m-1$.

(ii) If a complex polynomial differential equation of degree $m$ has $m+1$ real singular points, then it has the singular straight line $\bar{z}-z=0$. The proof of this well known result follows trivially from (21). Indeed, in this case differential equation (54) becomes

$$
\dot{z}=(\bar{z}-z) P(z, z, t) .
$$

(iii) If a complex polynomial differential equation of degree $m$ has $m+2$ real solutions, then we have

$$
\left|\begin{array}{cccccc}
1 & 1 & 1 & 1 & \ldots & 1 \\
z_{m+2} & z_{1} & z_{2} & z_{3} & \ldots & z_{m+1} \\
z_{m+2}^{2} & z_{1}^{2} & z_{2}^{2} & z_{3}^{2} & \ldots & z_{m+1}^{2} \\
\vdots & \vdots & \vdots & \vdots & \ldots & \vdots \\
z_{m+2}^{m} & z_{1}^{m} & z_{1}^{m} & z_{1}^{m} & \ldots & z_{m+1}^{m} \\
\dot{z}_{m+2} & \dot{z}_{1} & \dot{z}_{2} & \dot{z}_{3} & \ldots & \dot{z}_{m+1}
\end{array}\right|=0
$$

Indeed if $z=z_{m+2}(t)$ is another real solutions of a complex polynomial differential equation of degree $m$, then from (54) it follows (55). Consequently $z_{m+2}(t)$ is a solution of the generalized real Abel equation

$$
\dot{x}=\sum_{j=0}^{m} a_{j}(t) x^{j} .
$$

6.3. Polynomial differential system of degree $m>3$ with $m^{2}$ singular points. We shall determine the most general polynomial differential system of degree $m$ for which $\omega=\left\{\left(z_{1}, z_{2}, \ldots, z_{m^{2}}\right)\right\}$ where $z_{j}$ is constant for $j=1, \ldots, m^{2}$ such that $z_{j} \neq z_{k}$ for $j, k=$ $1, \ldots, m$ with $j \neq k$.

Proposition 19. The most general differential polynomial differential system of degree $m$ with $m^{2}$ singular points can be represented as follows

(56) $\dot{z}=\alpha_{11}(t) H_{11}(z, \bar{z}, t)+\alpha_{21}(t) H_{21}(z, \bar{z}, t)+\alpha_{22}(t) H_{22}(z, \bar{z}, t)+\ldots+\alpha_{\kappa \kappa}(t) H_{\kappa \kappa}(z, \bar{z}, t)$, 
where $a_{j n}(t)$ is an arbitrary function for $j, n=1, \ldots, \kappa>m$, and $H_{j n}(z, \bar{z}, t)$ for $j, n=$ $1, \ldots, \kappa$ are polynomials of degree $m$ elements of the $\kappa \times \kappa$ matrix $H=H_{m^{2}}$ where

$$
H_{r}=\left[H_{r-1}(z, \bar{z}, t), H_{r-1}\left(z_{r}, \bar{z}_{r}, t\right)\right]
$$

where $[H, G]=H G-G H$ is the Lie bracket, $r=1, \ldots, m^{2}$ and $H_{0}=G(z, \bar{z}, t)$ is an arbitrary $\kappa \times \kappa$ matrix whose are elements are arbitrary polynomials of degree $m$.

Proof. Clearly that all elements of the matrix $H_{1}=\left[H_{0}(z, \bar{z}, t), H_{0}\left(z_{1}, \bar{z}_{1}, t\right)\right]$ are vanishing when $z=z_{1}$, and all elements of the matrix

$$
H_{2}=\left[\left[H_{0}(z, \bar{z}, t), H_{0}\left(z_{1}, \bar{z}_{1}, t\right)\right],\left[H_{0}\left(z_{2}, \bar{z}_{2}, H_{0}\left(z_{1}, \bar{z}_{1}, t\right)\right]\right]\right.
$$

are vanishing when $z=z_{1}$, and $z=z_{2}$. By continuing this process we obtain that all elements of the matrix

$$
H_{m^{2}}=\left[H_{m^{2}-1}(z, \bar{z}, t), H_{m^{2}-1}\left(z_{m^{2}}, \bar{z}_{m^{2}}, t\right)\right],
$$

are vanishing when $z=z_{1}, \ldots, z=z_{m^{2}}$. Consequently the function

$$
\Phi=\alpha_{11}(t) H_{11}+\alpha_{12}(t) H_{12}+\alpha_{21}(t) H_{21}+\alpha_{22}(t) H_{22}+\ldots+\alpha_{\kappa \kappa}(t) H_{\kappa \kappa},
$$

is a polynomial of degree $m$ for which the given $m^{2}$ complex constants are it zeros. In short the proposition is proved.

Corollary 20. The most general differential polynomial differential system of degree $m$ with $m^{2}$ singular points can be represented as follows

$$
\dot{z}=<\tilde{\xi}(t), M(t) \xi^{T}>
$$

where $\xi=\left(1, z, \bar{z}, z^{2}, z \bar{z}^{2}, \bar{z}^{2}, \ldots, z^{m}, \ldots, \bar{z}^{m}\right), \tilde{\xi}(t)=\left.\xi\right|_{z=z_{m^{2}}(t), \bar{z}=\bar{z}_{m^{2}}(t)}$, and $M(t)$ is an arbitrary $(m+1)(m+2) / 2 \times(m+1)(m+2) / 2$ antisymmetric matrix such that $<\tilde{\xi}(t), M(t) \xi_{j}^{T}(t)>=$ 0 with $\xi_{j}=\left(1, z, \bar{z}_{j}, z_{j}^{2}, z \bar{z}_{j}^{2}, \bar{z}_{j}^{2}, \ldots, z_{j}^{m}, \ldots, \bar{z}_{j}^{m}\right)$, for $j=1, \ldots, m^{2}$.

Proof. After huge computations it is possible to show that there exists the antisymmetric matrix $M$ such that the right side of (56) can be written as

$$
\sum_{j, n=1}^{\kappa} \alpha_{j n} H_{j n}=<\tilde{\xi}(t), M(t) \xi^{T}>,
$$

where $H_{j n}$ are elements of the matrix $H_{m^{2}}$ given above.

\section{ACKNOWLEDGEMENTS}

The first author is partially supported by the Ministerio de Economía, Industria y Competitividad, Agencia Estatal de Investigación grant MTM2016-77278-P (FEDER), the Agència de Gestió d'Ajuts Universitaris i de Recerca grant 2017SGR1617, and the H2020 European Research Council grant MSCA-RISE-2017-777911.

\section{REFERENCES}

[1] T.A Alexeeva., V.F. Zaitsev and T.B. Shvets, On Discrete Symmetries of the Abel Equation of the second kind (in Russian), Applied Mechanics and Mathematics, MIDT, (1992), 4- 11.

[2] T.A Alexeeva., V.F. Zaitsev and T.B. Shvets, Handbook of Exact Solutions for Ordinary Differential Equations Chapman and Hall 1992

G. Alobaidi and R. Mailler, On the Abel equation of the second kind with sinusoidal forcing, Nonlinear Analysis: Modelling and Control 12 (2007), 33-44.

[3] L. Bougoffa, New exact general solutions of Abel equation of the second kind, Applied Mathematics and Computations 216 (2010), 689-691. 
[4] A.S. Galiullin, Inverse Problems of dynamics, Mir Publishers, 1984.

[5] G. Julia, Exercices d'Analyse, Tome III Équations Différentielles, Gauthier-Villars, Paris, (1933).

[6] Cima A., Gasull A. and Mañosas F. On the number of polynomial solutions of Bernoulli andAbel polynomial differential equations J. Differential Equations 263 (2017), 7099-7122.

[7] T. Harko And M.K. MAK, Relativistic dissipative cosmological models and Abel differential equations, Computer and Mathematics with applications 46 (2003), 849-853.

[8] H. Kinara, Five-dimensional monopole equation with hedgehog ansatz and Abel's differential equations, Phys. Rev. D 77 (2008), no. 12, 127703, 3 pp.

[9] J. P. M. Lebrun, On two coupled Abel type differential equations arising in a magnestatic problem, $I l$ Nuovo Cimento 103A (1990), 1369-1379.

[10] J. Llibre AND R. RAmíREz, Inverse problems in ordinary differential equations and applications, Progress in Math. 313, Birkhäuser, 2016.

[11] J. Llibre, R. Ramírez and N. Sadovskaia, On the 16th Hilbert problem for algebraic limit cycles, J. Differential Equations 248 (2010), 1401-1409.

[12] J. Llibre, R. Ramírez and N. Sadovskaia, On the 16th Hilbert problem for limit cycles on nonsingular algebric curves, J. Differential Equations 250 (2011), 983-999.

[13] J. Llibre, R. Ramírez and N. Sadovskaia, Integrability of the constrained rigid body, Nonlinear Dynamics 73 (2013), 2273-2290.

[14] J. Llibre, R. Ramírez, V. Ramírez and N. Sadovskaia, The 16th Hilbert problem restricted to circular algebraic limit cycles, J. Differential Equations 260 (2016), 5726-5760.

[15] J. Llibre, R. RamíRez and V. Ramírez, An inverse approach to the center-focus problem for polynomial differential system with homogenous nonlinearities, J. Differential Equations 263 (2017), 33273369 .

[16] J. Llibre, R. Ramírez and V. RamíRez, An inverse approach to the center problem, Rendiconti del Circolo Matematico di Palermo, II. Ser. 68 (2019), 29-64.

[17] J. Llibre, R. Ramírez and V. Ramírez, The center problem for the Lambda-Omega differential systems, to appear in J. Differential Equations, (2019).

[18] M.K. Mak and T. Harko, New Method for Generating General Solution of Abel Differential Equation, Computers and Mathematics with Applications 43 (2002), 91-94

[19] R.Muxarliamov, On the inverse problem in the qualitative theory of ordinary differential equations, Differencialnie yravnenuia 10 (1967) 1673-1681 (in Russian).

[20] N. Sadovskaia, Inverse problem in theory of ordinary differential equations. Thesis Ph. Dr., Univ. Politécnica de Cataluña, (2002) (in Spanish).

[21] X. Zhang, The 16th Hilbert problem on algebraic limit cycles, J. Differential Equations 251 (2011), $1778-1789$

1 Departament de Matemàtiques, Universitat Autònoma de Barcelona, 08193 Bellaterra, Barcelona, Catalonia, Spain.

Email address: jllibre@mat.uab.cat

2 Departament d'Enginyeria Informàtica i Matemàtiques, Universitat Rovira i Virgili, Avinguda dels Països Catalans 26, 43007 Tarragona, Catalonia, Spain.

Email address: rafaelorlando.ramirez@urv.cat

3 Departament de Matemàtica Aplicada II, Universitat Politècnica de Catalunya, C. Pau Gargallo 5, 08028 Barcelona, Catalonia, Spain.

Email address: natalia.sadovskaia@upc.edu 